University of Wollongong

Research Online

Faculty of Informatics - Papers (Archive)

Faculty of Engineering and Information

Sciences

$1-1-2007$

\title{
Performance improvement and dynamical behaviour analysis of a cascade of two CSTRs
}

Harvinder S. Sidhu

UNSW@ADFA, h.sidhu@adfa.edu.au

Simon D. Watt

UNSW@ADFA

Mark I. Nelson

University of Wollongong, mnelson@uow.edu.au

Ajay K. Ray

University of Western Ontario, aray@eng.uuw.ca

Follow this and additional works at: https://ro.uow.edu.au/infopapers

Part of the Physical Sciences and Mathematics Commons

\section{Recommended Citation}

Sidhu, Harvinder S.; Watt, Simon D.; Nelson, Mark I.; and Ray, Ajay K.: Performance improvement and dynamical behaviour analysis of a cascade of two CSTRs 2007, 1-13.

https://ro.uow.edu.au/infopapers/1652

Research Online is the open access institutional repository for the University of Wollongong. For further information contact the UOW Library: research-pubs@uow.edu.au 


\title{
Performance improvement and dynamical behaviour analysis of a cascade of two CSTRs
}

\begin{abstract}
We revisit the analysis of a reactor network consisting of two coupled continuous stirred tank reactors (CSTRs) arranged in series (Chem. Eng. J. 59 (1995) 169). The main idea proposed in this earlier work is to improve process conversion by generating periodic behaviour in the first reactor (by appropriate choice of design and operational parameters) which then 'forces' the second reactor. The performance of this cascade system was shown to be greatly enhanced using the above strategy. In this paper we show some conceptual errors in the analysis in the original paper. We also show that by employing a systematic bifurcation analysis, including the use of a path following software, greater insights can be gained regarding the system's behaviour. Using these techniques we show that operation and design parameters can be readily identified to ensure that the cascade has a superior performance to a single CSTR.
\end{abstract}

\section{Keywords}

Performance, Improvement, Dynamical, Behaviour, Analysis, Cascade, Two, CSTRs

\section{Disciplines}

Physical Sciences and Mathematics

\section{Publication Details}

Sidhu, H. S., Watt, S. D., Nelson, M. I. \& Ray, A. K. (2007). Performance improvement and dynamical behaviour analysis of a cascade of two CSTRs. International Journal of Chemical Reactor Engineering, 5 1-13. 


\title{
INTERNATIONAL JOURNAL OF CHEMICAL REACTOR ENGINEERING
}

\section{Performance Improvement and Dynamical Behaviour Analysis of a Cascade of Two CSTRs}

\author{
Harvinder S. Sidhu* \\ Simon D. Watt ${ }^{\dagger}$ \\ Mark I. Nelson $\ddagger$ \\ Ajay K. Ray**
}

*University of New South Wales at the Australian Defence Force Academy, h.sidhu@adfa.edu.au

${ }^{\dagger}$ University of New South Wales at the Australian Defence Force Academy, simon_watt@bigfoot.com

†University of Wollongong, nelsonm@member.ams.org

** University of Western Ontario, aray @eng.uwo.ca

ISSN 1542-6580 


\title{
Performance Improvement and Dynamical Behaviour Analysis of a Cascade of Two CSTRs*
}

\author{
Harvinder S. Sidhu, Simon D. Watt, Mark I. Nelson, and Ajay K. Ray
}

\begin{abstract}
We revisit the analysis of a reactor network consisting of two coupled continuous stirred tank reactors (CSTRs) arranged in series (Chem. Eng. J. 59 (1995) 169). The main idea proposed in this earlier work is to improve process conversion by generating periodic behaviour in the first reactor (by appropriate choice of design and operational parameters) which then 'forces' the second reactor. The performance of this cascade system was shown to be greatly enhanced using the above strategy. In this paper we show some conceptual errors in the analysis in the original paper. We also show that by employing a systematic bifurcation analysis, including the use of a path following software, greater insights can be gained regarding the system's behaviour. Using these techniques we show that operation and design parameters can be readily identified to ensure that the cascade has a superior performance to a single CSTR.
\end{abstract}

KEYWORDS: bifurcation, modelling, nonlinear dynamics, reaction engineering, stability, stirred-tank reactors, chemical oscillator

*This work was supported by a grant from the Australian Research Council (DP0559177). Comments by the referees are gratefully acknowledged. 


\section{INTRODUCTION}

Many studies, both experimental and theoretical, have shown that periodic forcing can be used to improve the conversion or selectivity of a desired product (Silveston et al., 1995 and Stankiewicz \& Kuczynski, 1995). However, the additional complications and costs associated with implementing these types of externally forced periodic operations have limited the industrial uptake of this technology (Silveston et al., 1995 and Stankiewicz \& Kuczynski, 1995).

The possibility of combining the advantages of periodic operation with the benefits of using two reactors arranged in series through the use of 'natural oscillations' has been investigated by several authors (Yang \& Su, 1993, Ray, 1995, Chen et al., 1995, Balakrishnan \& Yang, 1998, Jianqiang \& Ray, 2000 and Garhyan \& Elnashaie, 2004). By 'natural oscillations' it is meant that the operational parameters are chosen such that a steady feed of reactants into the first reactor produces self-sustained oscillations as its output which is then used to 'force' the second reactor. The main attraction of this method is that no external energy is required to generate oscillations, and consequently all the advantages of external forcing can be harnessed without the additional expense of implementing external forcing. Significant increases in product yields have been shown to be theoretically possible for various processes (Yang \& Su, 1993, Ray, 1995, Chen et al., 1995, Balakrishnan \& Yang, 1998, Jianqiang \& Ray, 2000 and Garhyan \& Elnashaie, 2004).

In this paper we re-investigate a cascade of two coupled non-isothermal continuously stirred tank reactors (CSTRs). In the original analysis (Ray, 1995), it was shown that process parameters can be chosen so that the output from the first reactor is oscillatory. This output acts as a forcing input for the second reactor. The conversion achieved by the cascade of two CSTRs was shown to be superior to that from a single CSTR with equal volume. We show that there are some conceptual errors in the bifurcation analysis presented in Ray (1995). We also show that the use of path following software provides greater insights to both the dynamical behaviour of the system and the performance of the system than direct integration of the governing equations. Important features, such as regions of bistability, may not be found when only direct integration of the governing equations is used. It was also shown that by extending the region of analysis, significant increases in the conversions are possible when the steady-state output from the first reactor creates a suitable condition by which the output from the second reactor is periodic - a situation which was not discussed in the earlier study.

Finally, we believe that this study helps to determine the conditions when oscillation occurs in a cascade of CSTRs, and when steady state non-oscillatory operation takes place. If an operator wants to avoid operating the system under oscillatory conditions, one should select the operating conditions appropriately. More importantly, this study enables one to determine if it is advantageous, in terms of performance improvement, to operate the system under oscillatory state over steady state operation for a specific reaction system. In addition, such an approach enables a convenient method to determine design and operating conditions for optimal reactor performance.

\section{MODEL DESCRIPTION AND GOVERNING EQUATIONS}

The chemical process is assumed to be an irreversible chemical reaction in which species $A$ converts to species $B$. This reaction occurs in a cascade of two CSTRs arranged in series. Following Ray (1995), we assume that there is no recycling and that the reaction is non-isothermal. The objective is to maximise the conversion of this reaction.

As discussed in Ray (1995), the dimensionless governing equations for the above system are given by 
Reactor 1:

$$
\begin{aligned}
& \frac{d X_{1}}{d t^{\prime}}=1-X_{1}\left[1+\alpha_{1} E\left(Y_{1}\right)\right] \\
& \frac{d Y_{1}}{d t^{\prime}}=-\left(1+\mu_{1}\right) Y_{1}+\alpha_{1} \beta_{1} X_{1} E\left(Y_{1}\right)
\end{aligned}
$$

Reactor 2 :

$$
\begin{aligned}
& \frac{d X_{2}}{d t^{\prime}}=\frac{V_{1}}{V_{2}}\left(X_{1}-X_{2}\left[1+\alpha_{2} E\left(Y_{2}\right)\right]\right) \\
& \frac{d Y_{2}}{d t^{\prime}}=\frac{V_{1}}{V_{2}}\left(\left(Y_{1}-Y_{F}\right) \times\left(\frac{T_{R_{1}}}{T_{R_{2}}}\right)^{2}-\left(1+\mu_{2}\right) Y_{2}+\alpha_{2} \beta_{2} X_{2} E\left(Y_{2}\right)\right)
\end{aligned}
$$

where the activation energy is given by

$$
E\left(Y_{i}\right)=\exp \left(\frac{Y_{i} \gamma_{i}}{Y_{i}+\gamma_{i}}\right), \quad i=1,2
$$

As in Ray (1995), we assume that the reactions are the same in both tanks and set the physical parameters to be equal, including the reference temperature, $T_{R_{i}}$, a weighted average of the temperature of the flow and the coolant.

\section{ONE TANK ANALYSIS}

Since there is no recycle in the system, it is possible to analyse the first tank independently of the second. A thorough analysis of the single reactor has been undertaken by Uppal et al. (1974), we report the main results from their study. The steady state solutions for the one tank are

$$
\begin{aligned}
X_{1}^{*} & =\frac{1}{1+\alpha_{1} E\left(Y_{1}^{*}\right)}, \\
Y_{1}^{*} & =\frac{\alpha_{1} \beta_{1} E\left(Y_{1}^{*}\right)}{\left[1+\mu_{1}\right]\left[1+\alpha_{1} E\left(Y_{1}^{*}\right)\right]} .
\end{aligned}
$$

Equation (6) can be re-arranged to give

$$
\alpha_{1}=\frac{Y_{1}^{*}}{\left(Q_{1}-Y_{1}^{*}\right) E\left(Y_{1}^{*}\right)},
$$

where $Q_{1}=\beta_{1} /\left(1+\mu_{1}\right)$. As stated in Uppal et al. (1974), this system will have a single steady state when

$$
Q_{1}<\frac{4 \gamma_{1}}{\gamma_{1}-4}
$$

Following Ray (1995), we focus our investigation to the case when there is a single steady state.

The stability of the steady-state solution is determined by linearizing about the equilibrium solution and is found to involve the growth function $e^{\lambda t}$, where the (possibly complex) constant $\lambda$ is given by the eigenvalues of the following Jacobian matrix

$$
J=\left(\begin{array}{cc}
-\left[1+\alpha_{1} E\left(Y_{1}^{*}\right)\right] & -\alpha_{1} X_{1}^{*} E\left(Y_{1}^{*}\right) \frac{\gamma_{1}^{2}}{\left(Y_{1}^{*}+\gamma_{1}\right)^{2}} \\
\alpha_{1} \beta_{1} E\left(Y_{1}^{*}\right) & -\left(1+\mu_{1}\right)+\alpha_{1} \beta_{1} X_{1}^{*} E\left(Y_{1}^{*}\right) \frac{\gamma_{1}^{2}}{\left(Y_{1}^{*}+\gamma_{1}\right)^{2}}
\end{array}\right)
$$

Since the design objective for the cascade is to generate oscillations in the first reactor, it is therefore essential to determine the conditions when periodic solutions are possible in the single CSTR system. In other words, we need to determine the locations of the Hopf bifurcation points. By the Hopf theorem (Seydel, 1994), the parameter values 
at which $\lambda$ becomes purely imaginary, are the points at which oscillatory solutions can be generated from the steady state solutions. This requires that the trace of the Jacobian matrix is zero and the determinant of the Jacobian matrix to be strictly positive. The trace of Jacobian vanishes when

$$
X_{1}^{*}=\frac{2+\mu_{1}+\alpha_{1} E\left(Y_{1}^{*}\right)}{\alpha_{1} \beta_{1} E\left(Y_{1}^{*}\right)} \frac{\left(\gamma_{1}+Y_{1}^{*}\right)^{2}}{\gamma_{1}^{2}} .
$$

Conditions (7) and (9) were used by Ray (1995) to "plot conversion in the first reactor for various values of the Damkohler number $\alpha_{1}$ or for different volumes $V_{1}$ of the first reactor when limit cycles exist".

Following Ray (1995), we plot the conversion in the single tank as a function of the Damkohler number $\alpha_{1}$ in Fig. 1. This figure is exactly the same as Fig. 2 in Ray (1995). However the interpretation given in Ray (1995) has some conceptual errors. The Hopf points only occur when the locus of the solution to equation (9) intersects the steady state solution (provided the determinant of the Jacobian matrix is positive). In Fig.1 there are two Hopf points at $\alpha_{1}=0.227$ and 0.621 . The lower Hopf point was found to be subcritical (unstable periodic solution emanating from the Hopf point) whereas the upper Hopf point is supercritical (stable solution emanating from it). Hence, there is a small region of bistability between $0.2217<\alpha_{1}<0.227$, where a stable steady state solution and a stable oscillatory solution exist. These results were obtained using the path following software AUTO (Doedel et al., 1998). Hence the periodic solution branch emanates from these two Hopf points (as will be demonstrated later in the manuscript). The trace $(J)=0$ locus does not give the conversion from periodic solutions. Condition (9), together with the steadystate conditions (5) and (6), only gives the locations of the Hopf bifurcation points on the steady-state curve. To obtain the conversion from the limit cycles, one needs to follow the branch of periodic solutions that emerge from the Hopf points. These are the main conceptual errors in Ray (1995).

Fig. 2 shows the case when the locus of the solution to equation (9) does not intersect the steady state solutions, and in this case there are no Hopf points for the given parameter values, and therefore the system does not exhibit oscillatory behaviour in this case. In Fig 3. of Ray (1995), the trace ( $\mathrm{J})=0$ locus was incorrectly plotted to show the conversion obtained by limit cycles, whereas there are no limit cycles in this case.

The Hopf curve in the $\left(Q_{1}, \alpha_{1}\right)$ - parameter plane is plotted in Fig. 3. The two points marked (A) represent the $\mathrm{H} 2{ }_{1}$ degeneracies (points where two Hopf points coalesce). Thus Hopf bifurcations do not occur if either the Damkohler number $\left(\alpha_{1}\right)$ is too large $\left(\alpha_{1}>0.61\right)$ or when the value of $Q_{1}$ is too low $\left(Q_{1}<2.41\right)$. Here we note that for the parameter values given in Fig. 2, $Q_{1}=2$ and therefore no Hopf points exist for this case, confirming the earlier results. We also note that one branch of the Hopf bifurcation locus ceases at the point labelled DZE at $\left(\alpha_{1}, Q_{1}\right)=(0.058,7.67)$. This point is the double-zero eigenvalue degeneracy point (or the Bogdanov-Takens bifurcation point) and beyond this point the condition that the determinant of the Jacobian matrix has to be positive fails. Hence for either $Q_{1}>7.67$ or $\alpha_{1}<0.058$, there is only one Hopf bifurcation point in the system.

\section{NUMERICS}

The path-following software AUTO (Doedel et al., 1998) was used to obtain steady-state diagrams. For a periodic orbit the norm that is used is the integral over the period of the periodic solution. Following Ray (1995) we investigate the percentage of conversion of $A$ to $B$ as a function of the volume of the first tank.

\section{$5 \quad$ RESULTS}

To investigate the improvement in the overall performance of the one and two tank models through natural oscillations, we consider the examples that were discussed in Ray (1995).

\section{$5.1 \quad$ Case 1}

We consider a first order irreversible exothermic chemical reaction in a non-isothermal CSTR. The parameters values used are based on those presented in Douglas \& Rippin (1966). The dimensionless parameters are

$$
\mu_{1}=\mu_{2}=5.0 ; \gamma_{1}=\gamma_{2}=35.332 ; \beta_{1}=\beta_{2}=15.547 ; Y_{F}=-4.326 .
$$




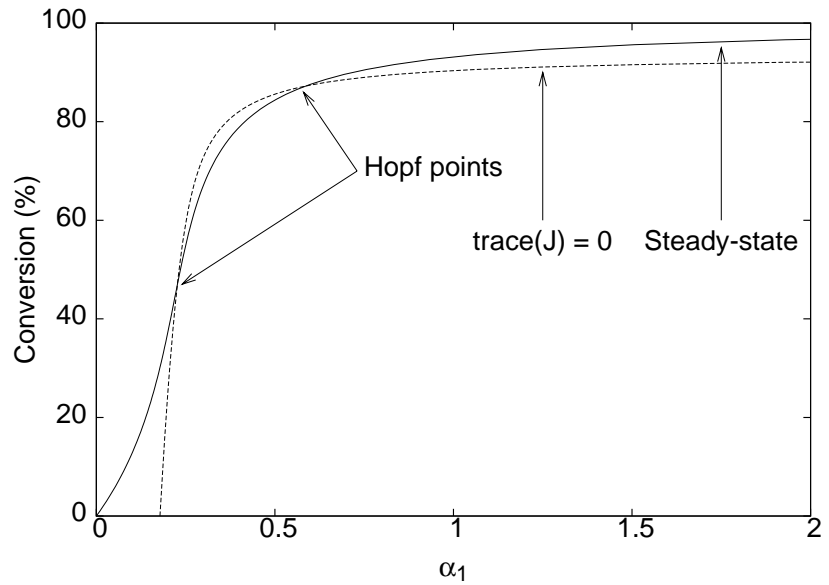

Figure 1: The steady state conversion for equations $(1-2)$ and the locus when trace $(\mathrm{J})=0$ for $\beta_{1}=18, \mu_{1}=5$ and $\gamma_{1}=40$. The intersections of these curves give the location of the Hopf points.

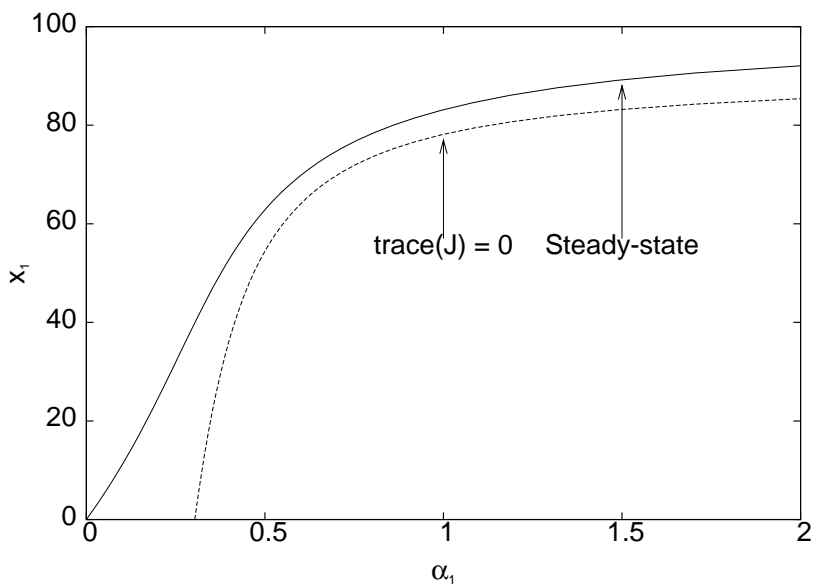

Figure 2: The steady state conversion for equations $(1-2)$ and the trace $(\mathrm{J})=0$ locus curve when $\beta_{1}=12, \mu_{1}=5$ and $\gamma_{1}=40$. 


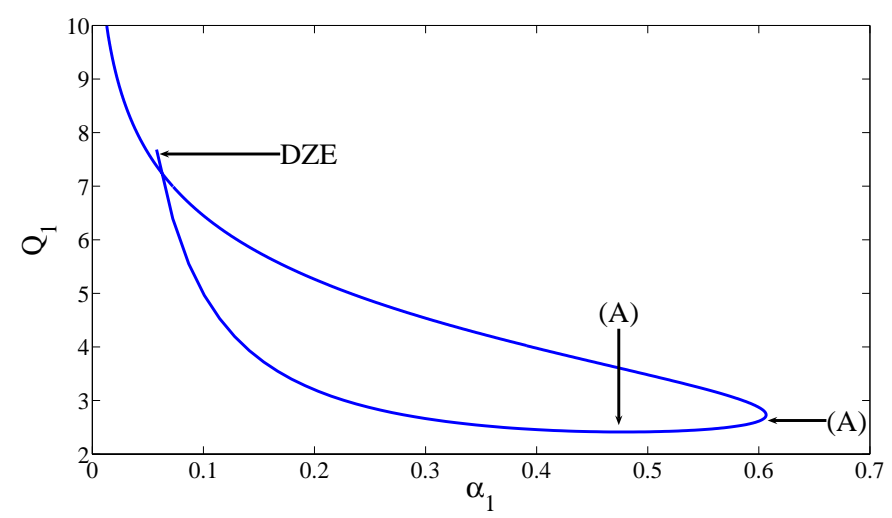

Figure 3: The Hopf point bifurcation locus in the $\left(\alpha_{1}, Q_{1}\right)$ - parameter plane for equations $(1-2)$. The points labelled (A) denote the $\mathrm{H} 2{ }_{1}$ degeneracies, whereas the point marked DZE represents the double-zero eigenvalue degenerate point.

The Damkohler number $\alpha_{1}$ is related to the volume of the first reactor $V_{1}$ by

$$
\alpha_{1}=3.33861 \times 10^{-4} \times V_{1} \text {. }
$$

Using these parameters, we find the locations of the Hopf points as $V_{1}=998 \mathrm{~m}^{3}$ and $V_{1}=1740 \mathrm{~m}^{3}$ : periodic solutions branch off from these points. Using AUTO, we find that there is a periodic solution branch connecting these two Hopf points, as shown in Fig. 4. In Ray (1995) it was stated that limit cycle behaviour does not occur when $V_{1}<615 \mathrm{~m}^{3}$, since the locus of the trace $(\mathrm{J})=0$ curve intersects the $V_{1}$ axis at $V_{1}=615 \mathrm{~m}^{3}$. As mentioned earlier, limit cycles only exists between the Hopf points mentioned above and nowhere else. As mentioned earlier this conceptual error is due to the fact that the Hopf points cannot be solely determined from the condition trace $(\mathrm{J})=0$.

From Fig. 4 we see that as the volume of the tank increases so does the conversion. We also note that the periodic solutions that exist between the Hopf points are stable and that the conversion obtained by operating the system in the oscillatory regime is only nominally higher than that of the unstable steady state. Unless a control scheme is implemented to enable the system to operate on the unstable steady state it is incorrect to compare the conversion between the stable periodic solution and the unstable steady-state (as was done in Ray, 1995), as the only global stable attractor between the two Hopf points is the limit cycle.

We now consider the case when a cascade of two CSTRs is utilized. Fig. 5 shows the conversion obtained for the cascade when the total volume of the two tanks is fixed at $2000 \mathrm{~m}^{3}$. From the single reactor analysis (Fig. 4), we know that there will be natural oscillations in the first tank when the volume of the first tank is between $998 \mathrm{~m}^{3}$ and $1740 \mathrm{~m}^{3}$. These oscillations will 'force' the second tank. We also know that in a single tank of volume $2000 \mathrm{~m}^{3}$ the conversion is $83.8 \%$.

From the figure we see that the maximum conversion of $92.1 \%$ is obtained when the volume of the first tank is made as small as possible, that is in the limit that $V_{1} \rightarrow 0$ (a degenerate case). This behavior is expected for reactions of this type (monotonically increasing), where a cascade will perform better if the smaller reactor is followed with the larger reactor (see e.g. Fogler, 1999). The conversion gradually drops as the volume of the first reactor is increased (or by reducing the volume of the second reactor) until the conversion reaches a local minimum of $84.1 \%$ when $V_{1}=918 \mathrm{~m}^{3}$. Further increase in $V_{1}$ results in the conversion increasing initially to a local maximum of $85.7 \%$ when $V_{1}=1140 \mathrm{~m}^{3}$ (noting that between $998<V_{1}<1740 \mathrm{~m}^{3}$ the global attractor is the limit cycle and therefore this maximum is obtained when the oscillations in the first reactor is forcing the second reactor), and then gradually decreasing to $83.8 \%$ at $V_{1}=2000 \mathrm{~m}^{3}$ which equals to the value of conversion for a single tank with the same total volume of $2000 \mathrm{~m}^{3}$ (this is again a degenerate case implying that the system is reduced to the single reactor case). Once again, in the absence of any control strategy that would allow the system to operate on the unstable steady state, 


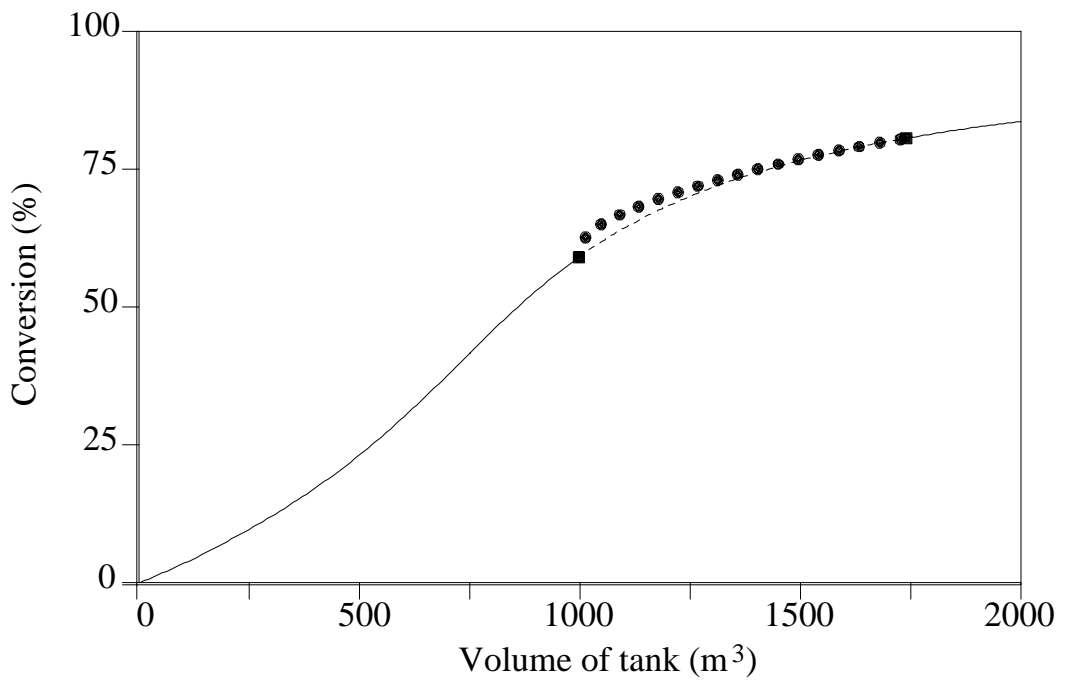

Figure 4: Conversion for a single tank versus the volume (Case 1). The solid lines represent stable steady states whereas the dashed line correspond to unstable steady-states. The squares are Hopf points and the solid circles represent the average conversions obtained from the stable periodic solutions.

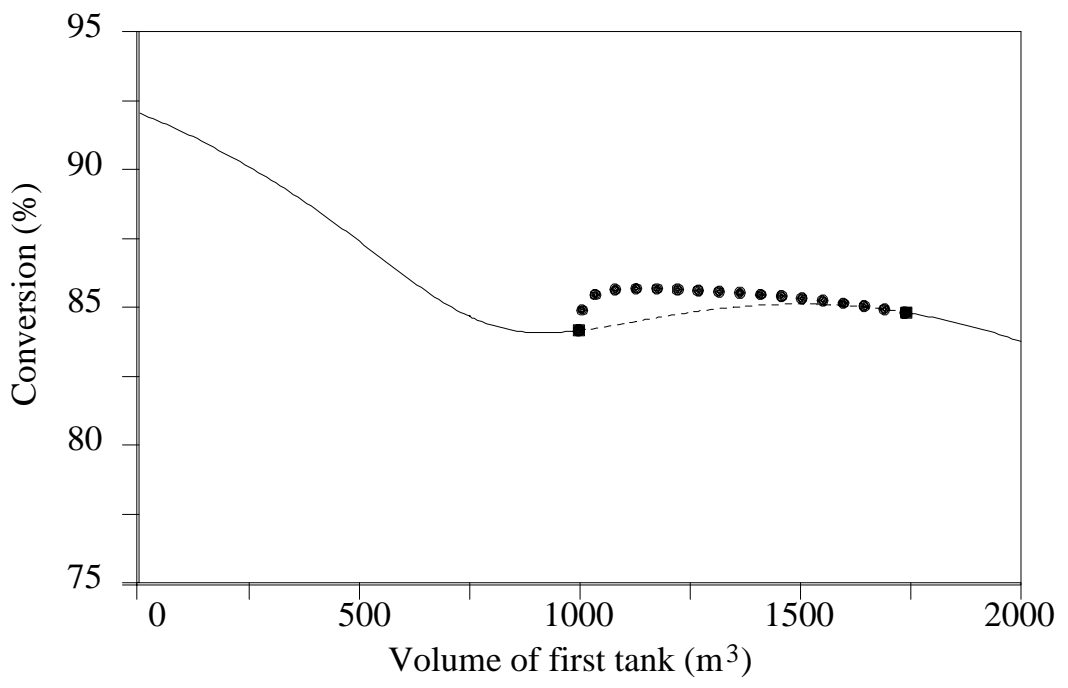

Figure 5: Conversion for two tanks in series versus the volume of the first tank (Case 1). The total volume of the cascade is $2000 \mathrm{~m}^{3}$. The notation used here is the same as Fig. 4. 
any comparisons made in Ray (1995) between the conversion obtained by the periodic solutions and the steady state solution between $998<V_{1}<1740 \mathrm{~m}^{3}$ is incorrect as there only exists one globally stable attractor.

From Figs. 4 and 5, we make the following conclusions for the case when the total volume of the two tanks is fixed at $2000 \mathrm{~m}^{3}$ : (i) the performance of the cascade configuration is always superior to the single tank case; (ii) the cascade performance is optimised by reducing the volume of the first tank to a value that is as small as possible and the operating conditions are such that there are no oscillations in the system; (iii) the optimal design conditions between $645<V_{1}<2000 \mathrm{~m}^{3}$ occurs when $V_{1}=1140 \mathrm{~m}^{3}$ (and $V_{2}=860 \mathrm{~m}^{3}$ ) at which natural oscillations from the output of the first tank forces the second reactor.

\subsection{Case 2}

The second example considered in Ray (1995) represents an industrial oxo reactor, as described by Vleeschhouwer \& Garton (1992). The reaction converts olefins and synthesis gas to aldehydes. The overall oxo reaction, with a fixed concentration of catalyst and synthesis gas, can be described by a first order irreversible reaction. For the set of parameter values stated in Ray (1995), the dimensionless parameters are

$$
\mu_{1}=\mu_{2}=9.766 ; \gamma_{1}=\gamma_{2}=25.474 ; \beta_{1}=\beta_{2}=30.524 ; Y_{F}=-7.5989 \text {. }
$$

The relationship between the Damkohler number $\alpha_{1}$ and the volume of the first reactor $V_{1}$ is

$$
\alpha_{1}=0.1071 \times V_{1}
$$

Fig. 6 shows the conversion obtained for the single tank case. Hopf points occur at $2.29 \mathrm{~m}^{3}$ and $9.94 \mathrm{~m}^{3}$. Unlike Case 1, the lower Hopf point is subcritical which results in an unstable periodic solution branch emanating from it which folds back and changes stability when the volume of the single tank is $2.23 \mathrm{~m}^{3}$. Hence when the volume of the reactor is between $2.23 \mathrm{~m}^{3}$ and $2.29 \mathrm{~m}^{3}$, there is a region of bistability when the solution can either converge to the stable steady state value (with a lower conversion), or onto the stable limit cycle (with a higher conversion). As also seen in Case 1, an increase in tank volume results in an increase in the overall conversion.

As in Ray (1995), we fix the total volume of the two reactors to be $3.5 \mathrm{~m}^{3}$, and vary the volume of the first reactor. The conversion achieved in a single tank with a volume of $3.5 \mathrm{~m}^{3}$ is $72.2 \%$. Fig. 7 shows the conversion obtained from the cascade. Although there are still two Hopf points for the system the periodic solution branches that emanates from them are not connected, as they are in Case 1. The upper Hopf point at $V_{1}=2.29 \mathrm{~m}^{3}$ is subcritical (an unstable periodic solution branch emanates from this Hopf point). The unstable periodic solution branch undergoes a fold bifurcation at $V_{1}=2.23 \mathrm{~m}^{3}$ changing stability, and the stable periodic solution persists up to $V_{1}=3.5 \mathrm{~m}^{3}$, at which point the two-reactor system reduces back to the one tank case. Hence for $2.23 \mathrm{~m}^{3}<V_{1}<2.29 \mathrm{~m}^{3}$ there is a region of bistability (higher conversion on the limit cycle, lower conversion on the stable steady state solution). The difference between the conversions of the limit cycle and steady state solutions in this region of bistability is around $11 \%$. The periodic solution that emerges from the upper Hopf point $V_{1}=2.29 \mathrm{~m}^{3}$ are the result of the Hopf bifurcation in the first reactor (see Fig. 6 for the single reactor analysis). On the other hand, the periodic solution that emerges from the lower Hopf point $V_{1}=1.29 \mathrm{~m}^{3}$ occurs when the output from the single reactor is non-oscillatory but the conditions in the second reactor are such that natural oscillations occur in this reactor.

As in Case 1, the maximum conversion, of $85.4 \%$, is obtained when the volume of the first tank is made as small as possible, that is in the limit as $V_{1} \rightarrow 0$ (degenerate case). However unlike in Case 1 , the high conversions that occur here are due to periodic solutions generated solely in the second reactor. (In Case 1, the high conversions for small $V_{1}$ were obtained through steady-state conditions in both reactors.) Since the investigation in Ray (1995) did not examine the behaviour of the system for values of $V_{1}$ smaller than $2.0 \mathrm{~m}^{3}$, this region of significant improvement in the system's conversion was not identified. The conversion gradually drops as the volume of the first reactor is increased until the conversion reaches a local minimum of $62.1 \%$ when $V_{1}=2.14 \mathrm{~m}^{3}$. Further increase in $V_{1}$ results in the conversion increasing to a local maximum of $72.2 \%$ when $V_{1}=3.5 \mathrm{~m}^{3}$, that is when the two-reactor cascade reduces back to the single CSTR case. Once again comparisons made in Ray (1995) (presented in Fig. 8 of that paper) between the conversions obtained by the stable periodic solutions and the unstable steady state solution are not appropriate. 
For Figs. 6 and 7 we make the following conclusions for the case when the total volume of the two tanks is fixed at $3.5 \mathrm{~m}^{3}$ : (i) for $0<V_{1}<1.29 \mathrm{~m}^{3}$, the two-reactor cascade is superior than the single tank solution and oscillatory solutions occur in the second reactor without being forced by the first reactor; (ii) for $1.29 \mathrm{~m}^{3}<V_{1}<3.5 \mathrm{~m}^{3}$, the single reactor of volume $3.5 \mathrm{~m}^{3}$ has a greater conversion than the cascade operating in a region with natural oscillations; (iii) both the single and cascade possess a narrow region of bistability - a higher conversion stable periodic solution, and a lower conversion stable steady-state solution.

\subsection{Futher analysis of Case 2}

In the previous section, we fixed the total volume of the system and determined the operational design parameters (the volume of the first tank) that gives the optimal conversion. In this section we further investigate the performance of the cascade and the single CSTR. We quantify the performance of these two systems by the total volume required to attain a specific conversion. The chosen conversion for comparison are $90 \%$ and $99 \%$. Fig. 8 shows the design parameters (values of $V_{1}$ and $V_{2}$ ) that are required to achieve the desired level of conversion, as well as the dynamic behaviour in each tank with such design parameters.

From Fig. 6, the volume needed to reach a $90 \%$ conversion in the single tank is $8.58 \mathrm{~m}^{3}$. In Fig. 8 this is denoted by the boundary of region $\mathrm{C}$ intersecting the horizontal axis. This conversion is achieved when the solution in the single tank is oscillatory as shown in Fig. 6. For the cascade, a steady-state diagram showing conversion as a function of the volume of the first tank when the total volume is greater than $3.5 \mathrm{~m}^{3}$ is qualitatively similar to that shown in Fig. 7, as the total volume increases, the entire conversion curve moves up and the lower Hopf point moves to the left, until it reaches the degenerate case $\left(V_{1} \rightarrow 0\right)$. The smallest total volume needed to achieve a conversion of $90 \%$ in the cascade is $4.74 \mathrm{~m}^{3}$, which corresponds to the degenerate case $V_{1} \rightarrow 0$. This is shown in Fig. 8 as the boundary of region A intersecting the vertical axis. As the volume in the second tank decreases, the volume in the first tank required to achieve a $90 \%$ conversion increases to $8.58 \mathrm{~m}^{3}$, the same as for a single CSTR (as discussed above). In the case of a cascade with equal volume tanks $\left(V_{1}=V_{2}\right), 90 \%$ conversion is achieved when the total volume of the cascade is $7.7 \mathrm{~m}^{3}$ (denoted by the point $P_{90}$ in Fig. 8). For such a cascade, it turns out that the total volume is $89.7 \%$ of that required for a single reactor.

Region $\mathrm{E}$ in Fig. 8 shows the values of $V_{1}$ and $V_{2}$ that are required to achieve a conversion of $99 \%$, or better. In the single CSTR, the volume of the tank that is required is $73.8 \mathrm{~m}^{3}$, whereas in the cascade, the minimal total volume needed is only $28.7 \mathrm{~m}^{3}$ (the volumes of the first and second tanks being $15.8 \mathrm{~m}^{3}$ and $12.9 \mathrm{~m}^{3}$ respectively). At $99 \%$ conversion, both the optimal single CSTR and the cascade operate at steady-state conditions. The total volume that is required for $99 \%$ conversion in the cascade when both tanks are of equal volumes $\left(V_{1}=V_{2}\right)$ is $28.94 \mathrm{~m}^{3}$ (denoted by the point $P_{99}$ in Fig. 8). This is only slightly larger than the optimal cascade design of $V_{1}=15.8 \mathrm{~m}^{3}$ and $V_{2}=12.9 \mathrm{~m}^{3}$ discussed above.

We have also briefly looked at the case of three equal volume reactors arranged in series. We found that to achieve a conversion of $90 \%$, the minimal total volume required is $7.65 \mathrm{~m}^{3}$ (compared to $8.58 \mathrm{~m}^{3}$ for a single reactor and $7.7 \mathrm{~m}^{3}$ for a double-reactor cascade) and to achieve a conversion of $99 \%$, the minimal total volume required for the three-reactor configuration is $24.18 \mathrm{~m}^{3}$ (compared to $73.8 \mathrm{~m}^{3}$ for a single reactor and $28.94 \mathrm{~m}^{3}$ for a double-reactor cascade). This suggests that although there could be further benefits by using a cascade of more than two reactors, the improvements in conversion of the three-tank cascade over the two-tank cascade are not as significant as those seen earlier for the single and double-reactor system. Furthermore one has to bear in mind the additional costs of including more reactors. 
(a)

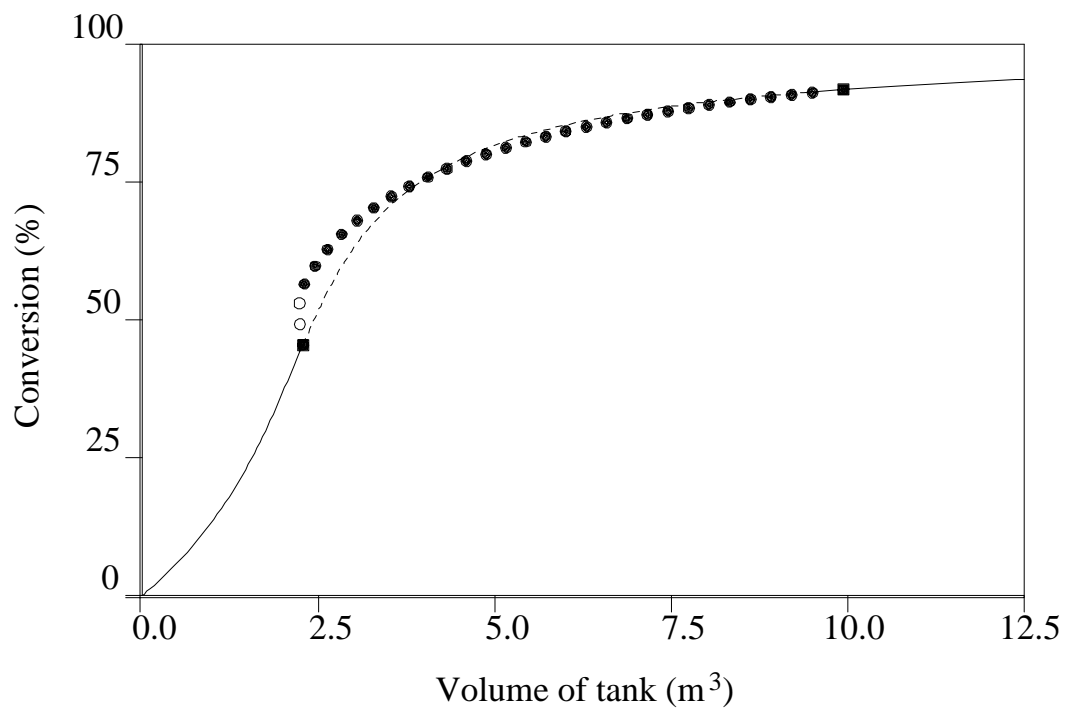

(b)

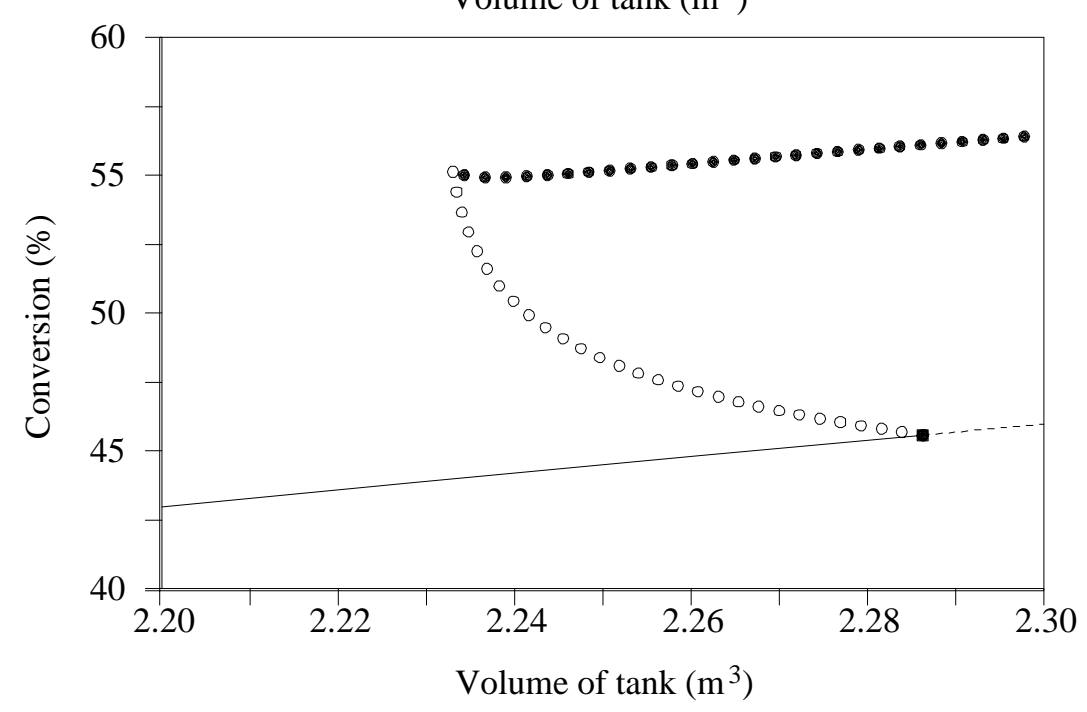

Figure 6: (a) Conversion for a single tank versus the volume (Case 2). The open circles denotes unstable oscillatory solutions, and the rest of the notation used here is similar to those used in Fig. 4. (b) Enlarged view of the narrow region of bistability that exists around the lower Hopf point. 
(a)

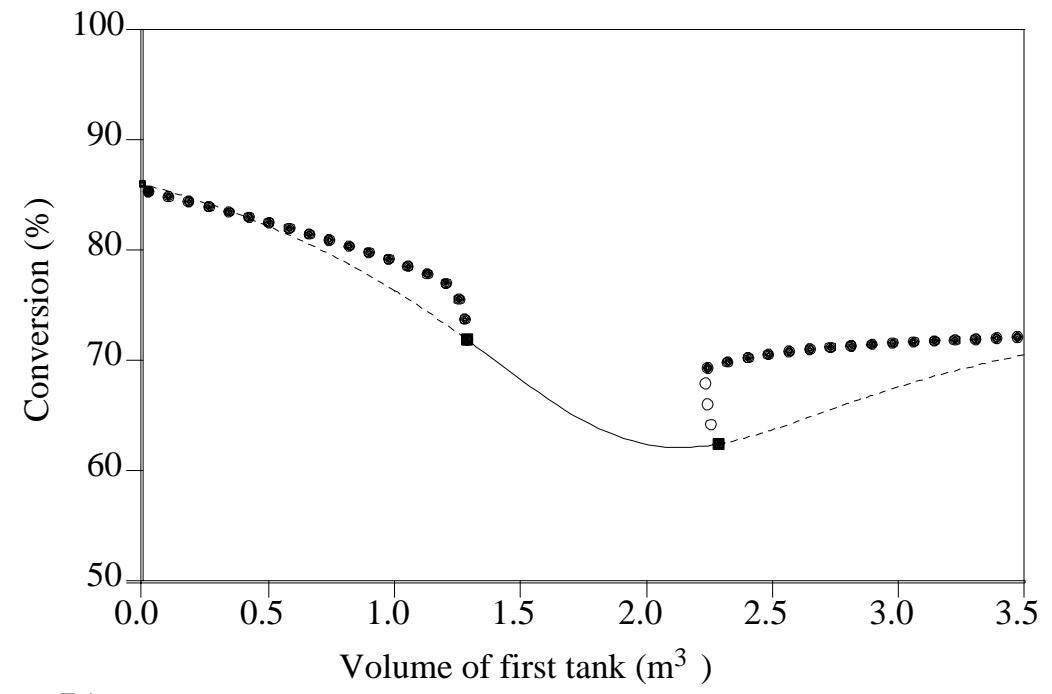

(b)

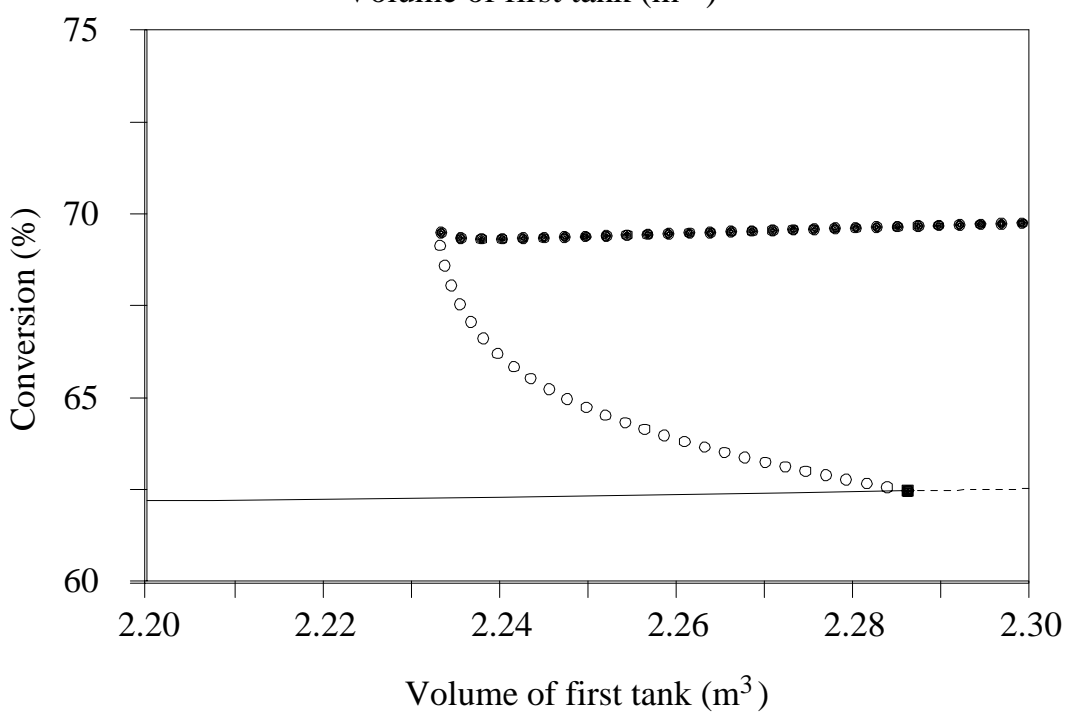

Figure 7: (a) Conversion for two tanks in series versus the volume of the first tank for Case 2. The total volume of the two tanks is $3.5 \mathrm{~m}^{3}$. The notation used here is the same as those used in Fig. 6. (b) Enlarged view of the narrow region of bistability that exists around the upper Hopf point. 


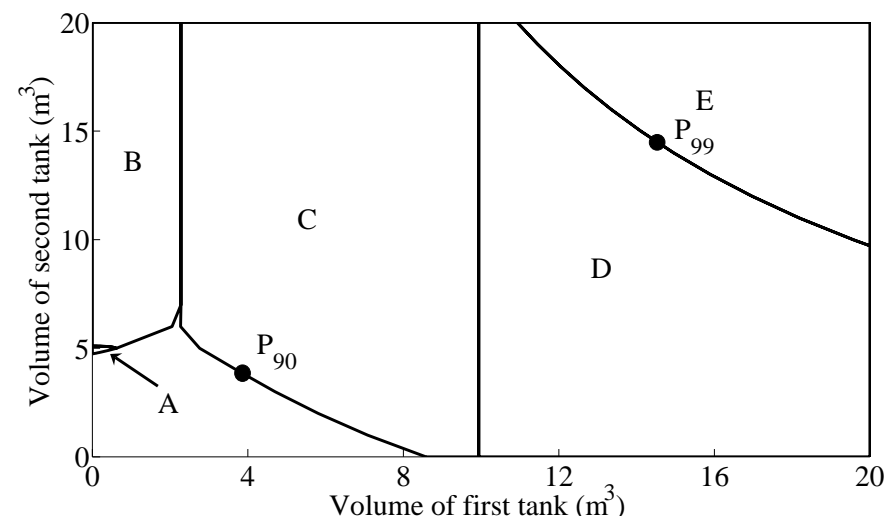

Figure 8: In regions A - D the conversion is at least $90 \%$ whereas in region $\mathrm{E}$ it is $99 \%$ or better. The dynamic behaviour in each region is : Region A - first tank in steady state and second tank oscillatory; Region B - first and second tank in steady state; Region C - first and second tank oscillatory (the first tank 'forcing' the second tank); Region D - first and second tank in steady state; Region E - first and second tank in steady state. Points $P_{90}$ and $P_{99}$ represents cascades with equal volume tanks $\left(V_{1}=V_{2}\right)$ that achieve $90 \%$ and $99 \%$ conversion respectively.

\section{CONCLUSIONS}

We have revisited the analysis of a two reactor cascade with no recycle for an irreversible exothermic reaction. Using the study reported in Ray (1995) as a basis, we used a systematic bifurcation analysis, including path-following software to analyse the behaviour of two systems. We showed that there were some conceptual errors in the dynamical systems analysis in Ray (1995). Furthermore we showed that operation and design parameters can be readily identified for optimal performance using the techniques outlined in this paper.

In the first example, we showed that the cascade configuration is always superior to the single tank, noting that the improvement in performance was achieved by designing the first reactor to be as small as possible. Furthermore, the improvement was achieved by operating the cascade at steady-state operating conditions, and not by use of using natural oscillations (which was the main thrust of the work outlined in Ray, 1995).

In the second example, the cascade was superior to the single tank. The best conversion again occurs when the volume of the first reactor is made as small as possible. However, unlike the previous example, in this case, the improvement is obtained by having a steady state condition in the first reactor and oscillatory solutions in this second reactor. This design strategy was not discussed in Ray (1995). Furthermore, we have also discovered regions of bistability with high and low conversions. Such bistability regions may not be observed if the only means of analysis is by direct integration, as was utilised in Ray (1995).

We further analysed the second example to compare the volume of tanks needed to achieve conversion of $90 \%$ and $99 \%$ in both the single CSTR and in the cascade. To achieve a conversion of $90 \%$, the cascade needed a total volume of about half that of the single CSTR. To obtain a conversion of $99 \%$, the total volume of the cascade is approximately a third required in a single CSTR. Such reductions in the total volume of the cascade over the single CSTR to achieve a given desired level of conversion may be a cost effective way of designing a reactor system. Preliminary analysis of the three-tank system suggests that although there maybe further benefits of including a cascade of more than two reactors, the improvement gained from such a system may not warrant the additional costs of including more than two tanks. 


\section{NOMENCLATURE}

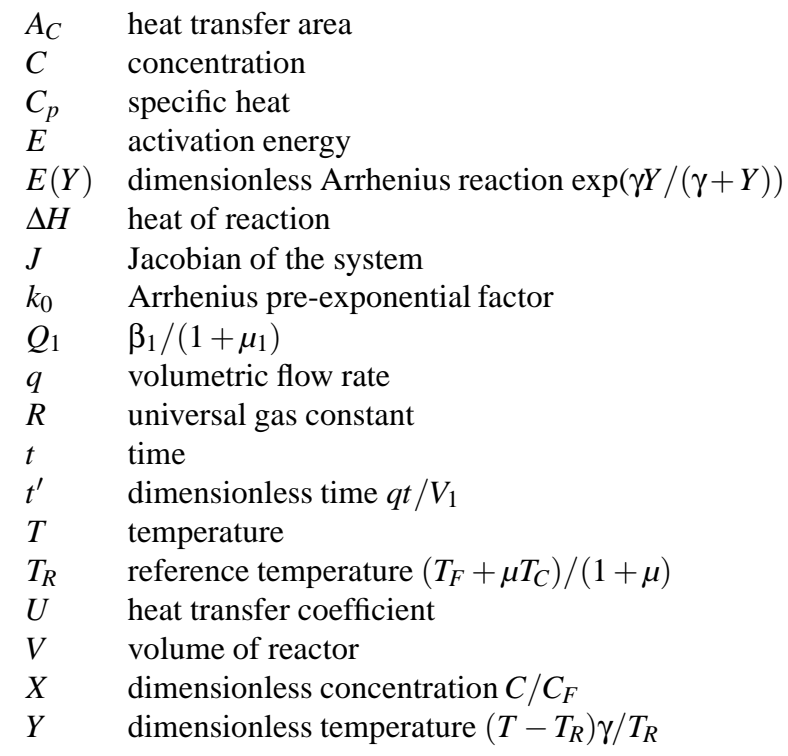

Greek letters

$\alpha \quad$ Damkohler number $\frac{V k_{0}}{q} \exp (-\gamma)$

$\beta \quad$ dimensionless adiabatic temperature rise $\frac{(-\Delta H) C_{F} \gamma}{\rho C_{p} T_{R}}$

$\gamma \quad$ dimensionless activation energy $E / R T_{R}$

$\mu \quad$ dimensionless heat transfer coefficient $U A_{C} / q \rho C_{p}$

$\rho$ density

\begin{tabular}{ll}
\multicolumn{2}{l}{ Subscripts } \\
1 & first reactor \\
2 & second reactor \\
C & coolant \\
F & feed
\end{tabular}

\section{REFERENCES}

Balakrishnan, A. and Yang, R.Y.K., "Improvement of chemostat performance via nonlinear oscillations Part 2: extension to other systems", ACH-Models in Chemistry, Vol. 135, 1-18 (1998).

Chen, C.C., Hwang, C. and Yang, R.Y.K., "Performance enhancement and optimization of chemostat cascades", Chemical Engineering Science, Vol. 50, No. 3, 485-494 (1995).

Doedel, E.J., Fairgrieve, T.F., Sandstede, B., Champneys, A.R., Kuznetsov, Y.A. and Wang, X., “Auto97: Continuation and bifurcation software for Ordinary Differential Equations (with Hom-Cont)". Available by anonymous ftp from ftp.cs. concordia.ca/pub/doedel/auto(1998).

Douglas, J.M. and Rippin, D.W.T., "Unsteady state process operation", Chemical Engineering Science, Vol. 21, No. 4, 305-315 (1966).

Fogler, H.S., "Elements of Chemical Engineering”, Upper Saddle River, N.J. : Prentice Hall PTR. (1999). 
Garhyan, P. and Elnashaie, S.S.E.H., "Bifurcation analysis of two continuous membrane fermentor configurations for producing ethanol”, Chemical Engineering Science, Vol. 59, 3235-3268 (2004).

Jianqiang, S. and Ray, A.K., "Performance improvement of activated sludge wastewater treatment by nonlinear natural oscillations", Chemical Engineering and Technology, Vol. 23, No. 12, 1115-1122 (2000).

Ray, A.K., "Performance improvement of a chemical reactor by non-linear natural oscillations", The Chemical Engineering Journal, Vol. 59, 169-175 (1995).

Seydel, R., "Practical Bifurcation and Stability Analysis: From Equilibrium to Chaos", Springer-Verlag, New York, (1994).

Silveston, P., Hudgins, R.R. and Renken, A., "Periodic operation of catalytic reactors - introduction and overview", Catalysis Today, Vol. 25, No. 2, 91-112 (1995).

Stankiewicz, A. and Kuczynski, M., "An industrial view on the dynamic operation of chemical converters", Chemical Engineering and Processing, Vol. 34,, No. 4, 367-377 (1995).

Uppal, A., Ray, W.H. and Poore, A.B., "On the dynamic behaviour of continuous stirred tank reactors", Chemical Engineering Science, Vol. 29, No. 4, 967-985 (1974).

Vleeschhouwer, P.H.M. and Garton, R.D., "Analysis of limit cycles in an industrial Oxo reactor", Chemical Engineering Science, Vol. 47, 2547-2552 (1992).

Yang, R.Y.K. and Su, J., "Improvement of chemostat performance via nonlinear oscillations Part 1: operation strategy", Bioprocess Engineering, Vol. 9, 97-102 (1993). 